\title{
A manifesto for the future of ICU trials
}

\author{
Ewan C. Goligher ${ }^{1,2,3^{*}}$ (D) Fernando Zampieri ${ }^{4}$, Carolyn S. Calfee ${ }^{5}$ and Christopher W. Seymour ${ }^{6}$
}

The intensive care unit (ICU) is both a challenging and opportune environment for the conduct of clinical trials. On the one hand, competing determinants of patient outcome (including multi-morbidity and pre-ICU illness trajectory) and the heterogeneity of critical illness syndromes attenuate the population-average treatment effect $[1,2]$. On the other hand, the ICU is a controlled environment that facilitates monitoring of protocol adherence and outcome ascertainment. ICU trials may be improperly powered because of overly optimistic assumptions about the baseline event rate in the control group and about the predicted effect of treatment on that event rate [3, 4]. The treatment effect required to demonstrate statistically significant benefit often substantially exceeds what might be considered the minimum clinically relevant benefit, and consequently, trials sometimes are interpreted to show "no evidence of benefit" even when clinically relevant benefits are observed.

The COVID-19 pandemic has shown that we need to (and can) find a way to deliver more effectively on trials in the ICU. The benefit of dexamethasone was demonstrated within just a few short months of the outbreak of the global pandemic [5]. Conversely, many tens of thousands of patients were treated with unproven and potentially harmful therapies outside of trials, and the benefit of certain interventions remains uncertain due to the challenges of completing trials of these rapidly adopted therapies.

We therefore propose a manifesto for the future of ICU trials (Table 1).
1 Think Bayesian Bayesian analysis is an alternate statistical paradigm that answers the question "what is the probability of treatment effect" in contrast to the traditional frequentist approach, which answers the question "what is the probability of these data, assuming no treatment effect?" Under the Bayesian framework, trial information is not biased by "looking at" the data, and the results can be continuously re-estimated and updated as additional information (i.e., patient outcomes) is added to the dataset [6]. To put it simply (and perhaps somewhat simplistically), conventional frequentist statistics views the entire trial as a single "coin flip"; technically, there is no information to draw conclusions until the trial is completed. By contrast, Bayesian statistics regards each individual patient's outcome as a "coin flip"; the estimated probability of benefit or harm can be continuously updated as information accumulates. We contend that the Bayesian approach is ideal because it (a) directly answers the questions of interest (probabilities of clinically relevant benefit, harm, or futility), thereby reducing the risk of a false "positive" or false "negative" conclusion; and (b) the continuously updated posterior permits maximally efficient trial adaptations in sample size and treatment allocation [7].

2 Adapt when needed Most trials in COVID-19 adopted an adaptive trial design given deep uncertainty about actual event rates and treatment effects. Adaptive designs respond flexibly to observed event rates and treatment effect, avoiding the risk of underestimating sample size requirement because of overly optimistic predictions about event rates and treatment effect [8]. Adapting treatment allocation probabilities within the randomization algorithm (response-adaptive randomization) can also increase trial efficiency in trials with three or more arms by

*Correspondence: ewan.goligher@uhn.ca

${ }^{3}$ Toronto General Hospital Research Institute, 585 University Ave., 11-PMB Room 192, Toronto, ON M5G 2N2, Canada

Full list of author information is available at the end of the article

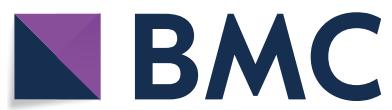

(c) The Author(s) 2020. Open Access This article is licensed under a Creative Commons Attribution 4.0 International License, which permits use, sharing, adaptation, distribution and reproduction in any medium or format, as long as you give appropriate credit to the original author(s) and the source, provide a link to the Creative Commons licence, and indicate if changes were made. The images or other third party material in this article are included in the article's Creative Commons licence, unless indicated otherwise in a credit line to the material. If material is not included in the article's Creative Commons licence and your intended use is not permitted by statutory regulation or exceeds the permitted use, you will need to obtain permission directly from the copyright holder. To view a copy of this licence, visit http://creativecommons.org/licenses/by/4.0/. The Creative Commons Public Domain Dedication waiver (http://creativeco mmons.org/publicdomain/zero/1.0/) applies to the data made available in this article, unless otherwise stated in a credit line to the data. 


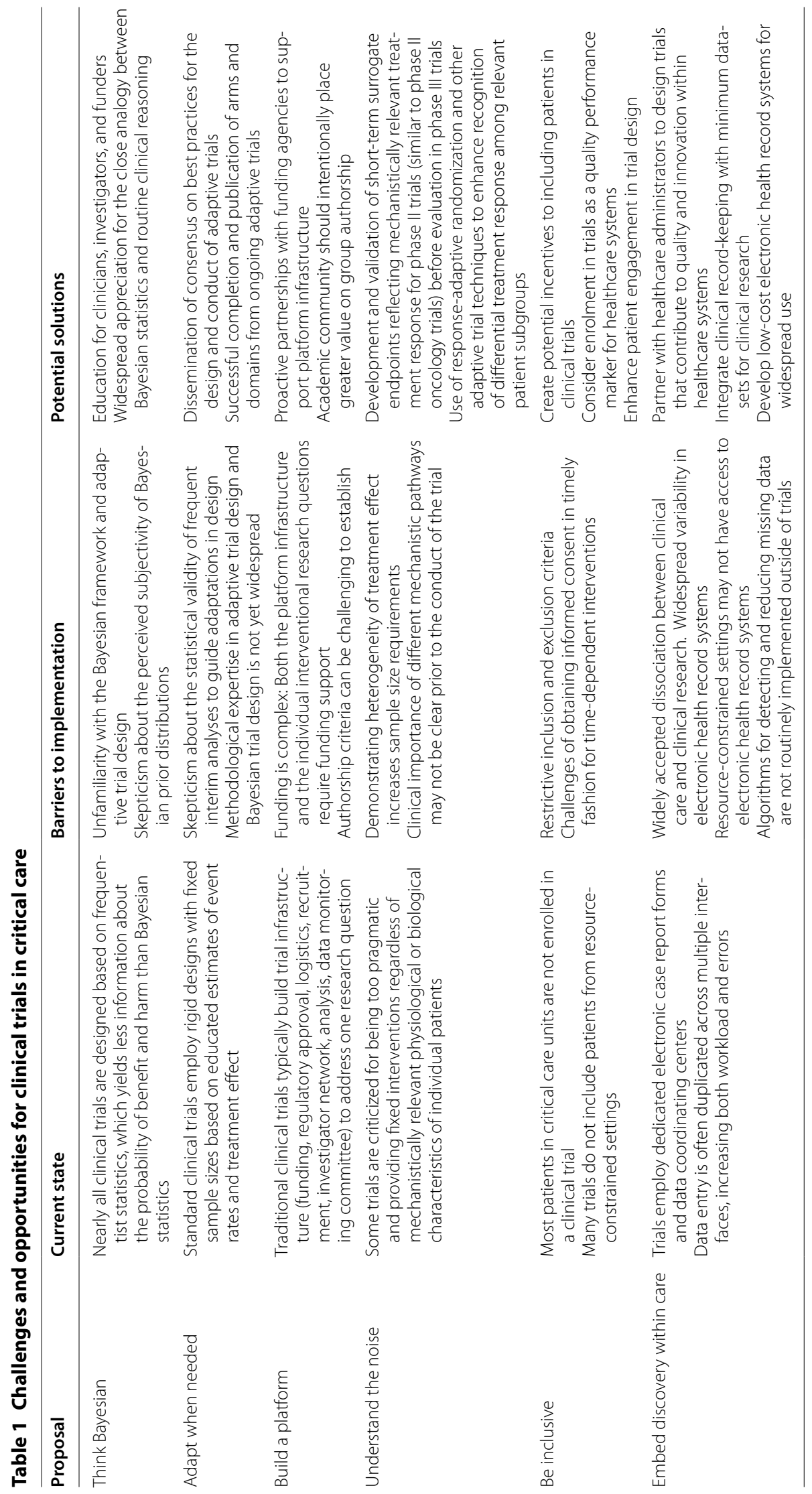


dropping poorly performing interventions and identifying treatment-responsive subgroups earlier (rather than waiting until the end of the trial to draw a conclusion).

3 Build a platform Platform trials leverage the infrastructure for recruitment, treatment allocation, outcome ascertainment, and analysis to evaluate multiple interventions for a single disease state or multiple disease states [8]. Just as "multiple games" can be played in a single "stadium," multiple trials-including Phase II, III, or IV trials-can be run sequentially or concurrently on a single platform. RECOVERY and REMAP-CAP provide important examples of phase III platform trials in COVID-19 [9, 10]. I-SPY2 provides a useful example of a Phase II platform intended to test many potential interventions for breast cancer, prioritizing the most promising agents for Phase III trials [11]. I-SPY2 investigators have now teamed with intensivists to launch an adaptive platform trial (I-SPY COVID) for severe COVID-19, with a similar Phase II focus.

4 Understand the noise In critical illness syndromes, the specific biological and physiological mechanisms driving outcomes may vary considerably ("noise") between patients. Hence, the benefit of therapeutics ("signal") targeting those mechanisms will also vary. To increase the probability of demonstrating the benefit of therapy in treatment-responsive subgroups (where such benefit actually exists) - to find the signal in the noise-heterogeneity in treatment response needs to be characterized as much as possible before and during Phase III trials [12, 13]. Adaptive trials can be designed to take account of relevant biological/physiological heterogeneity and to facilitate the discovery of such heterogeneity during the trial [11].

5 Be inclusive To ensure that trial results are truly generalizable, we need to ensure that appropriately diverse and representative patient populations are enrolled in clinical trials. Perhaps the best way to achieve this is to cast the widest possible net so that nearly every critically ill patient (including those outside traditional academic centers) has the opportunity to participate in a trial.

6 Embed discovery within care Trials are costly, data collection is labor-intensive, and finding patients can be difficult. Embedding trials-both electronically and culturally-offers a solution. Embedding trials within existing data repositories (e.g., clinical registries, electronic health records) to "find" patients, randomly assign treatments, collect data, and ascertain outcomes can increase efficiency and reduce costs. Trials can be embedded within the cul- ture of clinical practice to achieve continuous quality improvement through discovery and innovation, an approach referred to as "learning while doing" [14]. Utilizing the uncertainty of clinical decision-making as an opportunity for randomization could dramatically accelerate our capacity to improve care and outcomes for patients [15]. The culture of ICU healthcare delivery needs to increasingly see clinical trials as part of its core mission to deliver the very best possible care for patients. Trials are not ancillary to high-quality patient-centered care-they are integral to the mission.

\section{Acknowledgements \\ Not applicable.}

\section{Authors' contributions}

EG prepared the first draft and all authors critically revised the manuscript for intellectually important content. All authors read and approved the final manuscript.

\section{Funding}

E. Goligher is supported by an Early Career Investigator Award from the Canadian Institutes of Health Research.

\section{Availability of data and materials}

Not applicable.

\section{Ethics approval and consent to participate}

Not applicable.

\section{Consent for publication}

Not applicable.

\section{Competing interests}

Not applicable.

\section{Author details \\ ${ }^{1}$ Interdepartmental Division of Critical Care Medicine, University of Toronto, Toronto, Canada. ${ }^{2}$ Division of Respirology, Department of Medicine, University Health Network, Toronto, Canada. ${ }^{3}$ Toronto General Hospital Research Insti- tute, 585 University Ave., 11-PMB Room 192, Toronto, ON M5G 2N2, Canada. ${ }^{4}$ Research Institute, Hcor-Hospital Do Coração, São Paulo, Brazil. ${ }^{5}$ Division of Pulmonary, Critical Care, Allergy and Sleep Medicine, University of California San Francisco, San Francisco, CA, USA. ${ }^{6}$ The Clinical Research, Investigation, and Systems Modeling of Acute IIIness (CRISMA) Center, Department of Criti- cal Care Medicine, University of Pittsburgh School of Medicine, Pittsburgh, PA, USA.}

Received: 11 November 2020 Accepted: 16 November 2020

Published online: 09 December 2020

\section{References}

1. Iwashyna TJ, Burke JF, Sussman JB, Prescott HC, Hayward RA, Angus DC. Implications of heterogeneity of treatment effect for reporting and analysis of randomized trials in critical care. Am J Respir Crit Care Med. 2015;192(9):1045-51.

2. Seymour CW, Kennedy JN, Wang S, Chang CH, Elliott CF, Xu Z, Berry S, Clermont G, Cooper G, Gomez H, Huang DT, Kellum JA, Mi Q, Opal SM, Talisa V, van der Poll T, Visweswaran S, Vodovotz Y, Weiss JC, Yealy DM, Yende S, Angus DC. Derivation, validation, and potential treatment implications of novel clinical phenotypes for sepsis. JAMA J Am Med Assoc. 2019;321(20):2003-17. 
3. Abrams D, Montesi SB, Moore SKL, Manson DK, Klipper KM, Case MA, Brodie D, Beitler JR. Powering bias and clinically important treatment effects in randomized trials of critical illness. Crit Care Med. 2020;48(12):1710-9.

4. Harhay MO, Wagner J, Ratcliffe SJ, Bronheim RS, Gopal A, Green S, Cooney E, Mikkelsen ME, Kerlin MP, Small DS, Halpern SD. Outcomes and statistical power in adult critical care randomized trials. Am J Respir Crit Care Med. 2014;189(12):1469-78.

5. RECOVERY Collaborative Group, Horby P, Lim WS, Emberson JR, Mafham M, Bell JL, Linsell L, Staplin N, Brightling C, Ustianowski A, Elmahi E, Prudon B, Green C, Felton T, Chadwick D, Rege K, Fegan C, Chappell LC, Faust SN, Jaki T, Jeffery K, Montgomery A, Rowan K, Juszczak E, Baillie JK, Haynes R, Landray MJ. Dexamethasone in hospitalized patients with Covid-19preliminary report. N Engl J Med. 2020;17:NEJMoa2021436. https://doi. org/10.1056/NEJMoa2021436.

6. Berry DA. Bayesian clinical trials. Nat Rev Drug Discov. 2006;5(1):27-36.

7. Seymour CW, McCreary EK, Stegenga J. Sensible medicine-balancing intervention and inaction during the COVID-19 pandemic. JAMA. 2020. https://doi.org/10.1001/jama.2020.20271.

8. Angus DC, Alexander BM, Berry S, Buxton M, Lewis R, Paoloni M, Webb SAR, Arnold S, Barker A, Berry DA, Bonten MJM, Brophy M, Butler C, Cloughesy TF, Derde LPG, Esserman LJ, Ferguson R, Fiore L, Gaffey SC, Gaziano JM, Giusti K, Goossens H, Heritier S, Hyman B, Krams M, Larholt K, LaVange LM, Lavori P, Lo AW, London AJ, Manax V, McArthur C, O'Neill G, Parmigiani G, Perlmutter J, Petzold EA, Ritchie C, Rowan KM, Seymour CW, Shapiro N, Simeone DM, Smith B, Spellberg B, Stern AD, Trippa L, Trusheim M, Viele K, Wen PY, Woodcock J. Adaptive platform trials: definition, design, conduct and reporting considerations. Nat Rev Drug Discov. 2019;18(10):797-807.

9. Normand ST. The RECOVERY Platform. N Engl J Med. 2020. https://doi. org/10.1056/NEJMe2025674.

10. Angus DC, Derde L, Al-Beidh F, Annane D, Arabi Y, Beane A, van Bentum-Puijk W, Berry L, Bhimani Z, Bonten M, Bradbury C, Brunkhorst F, Buxton M, Buzgau A, Cheng AC, de Jong M, Detry M, Estcourt L, Fitzgerald M, Goossens H, Green C, Haniffa R, Higgins AM, Horvat C, Hullegie S, Kruger P, Lamontagne F, Lawler PR, Linstrum K, Litton E, Lorenzi E, Marshall J, McAuley D, McGlothin A, McGuinness S, McVerry B, Montgomery S, Mouncey P, Murthy S, Nichol A, Parke R, Parker J, Rowan K, Sanil A, Santos M, Saunders C, Seymour C, Turner A, van de Veerdonk F, Venkatesh B, Zarychanski R, Berry S, Lewis RJ, McArthur C, Webb SA, Gordon AC; Writing Committee for the REMAP-CAP Investigators, Al-Beidh F, Angus D, Annane D, Arabi Y, van Bentum-Puijk W, Berry S, Beane A, Bhimani Z, Bonten M, Bradbury C, Brunkhorst F, Buxton M, Cheng A, De Jong M, Derde L, Estcourt L, Goossens H, Gordon A, Green C, Haniffa R, Lamontagne F, Lawler P, Litton E, Marshall J, McArthur C, McAuley D, McGuinness S, McVerry B, Montgomery S, Mouncey P, Murthy S, Nichol A, Parke R, Rowan K, Seymour C, Turner A, van de Veerdonk F, Webb S, Zarychanski R, Campbell L, Forbes A, Gattas D, Heritier S, Higgins L, Kruger P, Peake S, Presneill J, Seppelt I, Trapani T, Young P, Bagshaw S, Daneman N, Ferguson N, Misak C, Santos M, Hullegie S, Pletz M, Rohde G, Rowan K, Alexander B, Basile K, Girard T, Horvat C, Huang D, Linstrum K, Vates J, Beasley R, Fowler R, McGloughlin S, Morpeth S, Paterson D, Venkatesh B, Uyeki T, Baillie K, Duffy E, Fowler R, Hills T, Orr K, Patanwala A, Tong S, Netea M, Bihari S, Carrier M, Fergusson D, Goligher E, Haidar G, Hunt B, Kumar A, Laffan M, Lawless P, Lother S, McCallum P, Middeldopr S, McQuilten Z, Neal M, Pasi J, Schutgens R, Stanworth S, Turgeon A, Weissman A, Adhikari N, Anstey M, Brant E, de Man A, Lamonagne F, Masse MH, Udy A, Arnold D, Begin P, Charlewood R, Chasse M, Coyne M, Cooper J, Daly J, Gosbell I, Harvala-Simmonds H, Hills T, MacLennan S, Menon D, McDyer J, Pridee N, Roberts D, Shankar-Hari M, Thomas H, Tinmouth A, Triulzi D, Walsh T, Wood E, Calfee C, O'Kane C, Shyamsundar M, Sinha P, Thompson T, Young I, Bihari S, Hodgson C, Laffey J, McAuley D, Orford N, Neto A, Detry M, Fitzgerald M, Lewis R, McGlothlin A, Sanil A, Saunders C, Berry L, Lorenzi E, Miller E, Singh V, Zammit C, van Bentum Puijk W, Bouwman W, Mangindaan Y, Parker L, Peters S, Rietveld I, Raymakers K, Ganpat R, Brillinger N, Markgraf R, Ainscough K, Brickell K, Anjum A, Lane JB, Richards-Belle A, Saull M, Wiley D, Bion J, Connor J, Gates S, Manax V, van der Poll T, Reynolds J, van Beurden M, Effelaar E, Schotsman J, Boyd C, Harland C, Shearer A, Wren J, Clermont G, Garrard W, Kalchthaler K, King A, Ricketts D, Malakoutis S, Marroquin O, Music E, Quinn K, Cate H, Pearson K, Collins J, Hanson J, Williams P, Jackson S, Asghar A, Dyas S, Sutu M, Murphy S, Williamson D, Mguni N, Potter A,
Porter D, Goodwin J, Rook C, Harrison S, Williams H, Campbell H, Lomme K, Williamson J, Sheffield J, van't Hoff W, McCracken P, Young M, Board J, Mart E, Knott C, Smith J, Boschert C, Affleck J, Ramanan M, D'Souza R, Pateman K, Shakih A, Cheung W, Kol M, Wong H, Shah A, Wagh A, Simpson J, Duke G, Chan P, Cartner B, Hunter S, Laver R, Shrestha T, Regli A, Pellicano A, McCullough J, Tallott M, Kumar N, Panwar R, Brinkerhoff G, Koppen C, Cazzola F, Brain M, Mineall S, Fischer R, Biradar V, Soar N, White H, Estensen K, Morrison L, Smith J, Cooper M, Health M, Shehabi Y, Al-Bassam W, Hulley A, Whitehead C, Lowrey J, Gresha R, Walsham J, Meyer J, Harward M, Venz E, Williams P, Kurenda C, Smith K, Smith M, Garcia R, Barge D, Byrne D, Byrne K, Driscoll A, Fortune L, Janin P, Yarad E, Hammond N, Bass F, Ashelford A, Waterson S, Wedd S, McNamara R, Buhr H, Coles J, Schweikert S, Wibrow B, Rauniyar R, Myers E, Fysh E, Dawda A, Mevavala B, Litton E, Ferrier J, Nair P, Buscher H, Reynolds C, Santamaria J, Barbazza L, Homes J, Smith R, Murray L, Brailsford J, Forbes L, Maguire T, Mariappa V, Smith J, Simpson S, Maiden M, Bone A, Horton M, Salerno T, Sterba M, Geng W, Depuydt P, De Waele J, De Bus L, Fierens J, Bracke S, Reeve B, Dechert W, Chassé M, Carrier FM, Boumahni D, Benettaib F, Ghamraoui A, Bellemare D, Cloutier Ė, Francoeur C, Lamontagne F, D'Aragon F, Carbonneau E, Leblond J, Vazquez-Grande G, Marten N, Wilson M, Albert M, Serri K, Cavayas A, Duplaix M, Williams V, Rochwerg B, Karachi T, Oczkowski S, Centofanti J, Millen T, Duan E, Tsang J, Patterson L, English S, Watpool I, Porteous R, Miezitis S, McIntyre L, Brochard L, Burns K, Sandhu G, Khalid I, Binnie A, Powell E, McMillan A, LukT, Aref N, Andric Z, Cviljevic S, Đimoti R, Zapalac M, Mirković G, Baršić B, Kutleša M, Kotarski V, Vujaklija Brajković A, Babel J, Sever H, Dragija L, Kušan I, Vaara S, Pettilä L, Heinonen J, Kuitunen A, Karlsson S, Vahtera A, Kiiski H, Ristimäki S, Azaiz A, Charron C, Godement M, Geri G, Vieillard-Baron A, Pourcine F, Monchi M, Luis D, Mercier R, Sagnier A, Verrier N, Caplin C, Siami S, Aparicio C, Vautier S, Jeblaoui A, Fartoukh M, Courtin L, Labbe V, Leparco C, Muller G, Nay MA, Kamel T, Benzekri D, Jacquier S, Mercier E, Chartier D, Salmon C, Dequin P, Schneider F, Morel G, L'Hotellier S, Badie J, Berdaguer FD, Malfroy S, Mezher C, Bourgoin C, Megarbane B, Voicu S, Deye N, Malissin I, Sutterlin L, Guitton C, Darreau C, Landais M, Chudeau N, Robert A, Moine P, Heming N, Maxime V, Bossard I, Nicholier TB, Colin G, Zinzoni V, Maquigneau N, Finn A, Kreß G, Hoff U, Friedrich Hinrichs C, Nee J, Pletz M, Hagel S, Ankert J, Kolanos S, Bloos F, Petros S, Pasieka B, Kunz K, Appelt P, Schütze B, Kluge S, Nierhaus A, Jarczak D, Roedl K, Weismann D, Frey A, Klinikum Neukölln V, Reill L, Distler M, Maselli A, Bélteczki J, Magyar I, Fazekas Á, Kovács S, Szőke V, Szigligeti G, Leszkoven J, Collins D, Breen P, Frohlich S, Whelan R, McNicholas B, Scully M, Casey S, Kernan M, Doran P, O'Dywer M, Smyth M, Hayes L, Hoiting O, Peters M, Rengers E, Evers M, Prinssen A, Bosch Ziekenhuis J, Simons K, Rozendaal W, Polderman F, de Jager P, Moviat M, Paling A, Salet A, Rademaker E, Peters AL, de Jonge E, Wigbers J, Guilder E, Butler M, Cowdrey KA, Newby L, Chen Y, Simmonds C, McConnochie R, Ritzema Carter J, Henderson S, Van Der Heyden K, Mehrtens J, Williams T, Kazemi A, Song R, Lai V, Girijadevi D, Everitt R, Russell R, Hacking D, Buehner U, Williams E, Browne T, Grimwade K, Goodson J, Keet O, Callender O, Martynoga R, Trask K, Butler A, Schischka L, Young C, Lesona E, Olatunji S, Robertson Y, José N, Amaro dos Santos Catorze T, de Lima Pereira TNA, Neves Pessoa LM, Castro Ferreira RM, Pereira Sousa Bastos JM, Aysel Florescu S, Stanciu D, Zaharia MF, Kosa AG, Codreanu D, Marabi Y, Al Qasim E, Moneer Hagazy M, Al Swaidan L, Arishi H, Muñoz-Bermúdez R, Marin-Corral J, Salazar Degracia A, Parrilla Gómez F, Mateo López MI, Rodriguez Fernandez J, Cárcel Fernández S, Carmona Flores R, León López R, de la Fuente Martos C, Allan A, Polgarova P, Farahi N, McWilliam S, Hawcutt D, Rad L, O'Malley L, Whitbread J, Kelsall O, Wild L, Thrush J, Wood H, Austin K, Donnelly A, Kelly M, O'Kane S, McClintock D, Warnock M, Johnston P, Gallagher LJ, Mc Goldrick C, Mc Master M, Strzelecka A, Jha R, Kalogirou M, Ellis C, Krishnamurthy V, Deelchand V, Silversides J, McGuigan P, Ward K, O'Neill A, Finn S, Phillips B, Mullan D, Oritz-Ruiz de Gordoa L, Thomas M, Sweet K, Grimmer L, Johnson R, Pinnell J, Robinson M, Gledhill L, Wood T, Morgan M, Cole J, Hill H, Davies M, Antcliffe D, Templeton M, Rojo R, Coghlan P, Smee J, Mackay E, Cort J, Whileman A, Spencer T, Spittle N, Kasipandian V, Patel A, Allibone S, Genetu RM, Ramali M, Ghosh A, Bamford P, London E, Cawley K, Faulkner M, Jeffrey H, Smith T, Brewer C, Gregory J, Limb J, Cowton A, O'Brien J, Nikitas N, Wells C, Lankester L, Pulletz M, Williams P, Birch J, Wiseman S, Horton S, Alegria A, Turki S, Elsefi T, Crisp N, Allen L, McCullagh I, Robinson P, Hays C, Babio-Galan M, Stevenson H, Khare D, Pinder M, Selvamoni S, Gopinath A, Pugh R, Menzies D, Mackay C, Allan E, Davies G, Puxty K, 
McCue C, Cathcart S, Hickey N, Ireland J, Yusuff H, Isgro G, Brightling C, Bourne M, Craner M, Watters M, Prout R, Davies L, Pegler S, Kyeremeh L, Arbane G, Wilson K, Gomm L, Francia F, Brett S, Sousa Arias S, Elin Hall R, Budd J, Small C, Birch J, Collins E, Henning J, Bonner S, Hugill K, Cirstea E, Wilkinson D, Karlikowski M, Sutherland H, Wilhelmsen E, Woods J, North J, Sundaran D, Hollos L, Coburn S, Walsh J, Turns M, Hopkins P, Smith J, Noble H, Depante MT, Clarey E, Laha S, Verlander M, Williams A, Huckle A, Hall A, Cooke J, Gardiner-Hill C, Maloney C, Qureshi H, Flint N, Nicholson S, Southin S, Nicholson A, Borgatta B, Turner-Bone I, Reddy A, Wilding L, Chamara Warnapura L, Agno Sathianathan R, Golden D, Hart C, Jones J, Bannard-Smith J, Henry J, Birchall K, Pomeroy F, Quayle R, Makowski A, Misztal B, Ahmed I, KyereDiabour T, Naiker K, Stewart R, Mwaura E, Mew L, Wren L, Willams F, Innes R, Doble P, Hutter J, Shovelton C, Plumb B, Szakmany T, Hamlyn V, Hawkins N, Lewis S, Dell A, Gopal S, Ganguly S, Smallwood A, Harris N, Metherell S, Lazaro JM, Newman T, Fletcher S, Nortje J, Fottrell-Gould D, Randell G, Zaman M, Elmahi E, Jones A, Hall K, Mills G, Ryalls K, Bowler H, Sall J, Bourne R, Borrill Z, Duncan T, Lamb T, Shaw J, Fox C, Moreno Cuesta J, Xavier K, Purohit D, Elhassan M, Bakthavatsalam D, Rowland M, Hutton P, Bashyal A, Davidson N, Hird C, Chhablani M, Phalod G, Kirkby A, Archer S, Netherton K, Reschreiter H, Camsooksai J, Patch S, Jenkins S, Pogson D, Rose S, Daly Z, Brimfield L, Claridge H, Parekh D, Bergin C, Bates M, Dasgin J, McGhee C, Sim M, Hay SK, Henderson S, Phull MK, Zaidi A, Pogreban T, Rosaroso LP, Harvey D, Lowe B, Meredith M, Ryan L, Hormis A, Walker R, Collier D, Kimpton S, Oakley S, Rooney K, Rodden N, Hughes E, Thomson N, McGlynn D, Walden A, Jacques N, Coles H, Tilney E, Vowell E, Schuster-Bruce M, Pitts S, Miln R, Purandare L, Vamplew L, Spivey M, Bean S, Burt K, Moore L, Day C, Gibson C, Gordon E, Zitter L, Keenan S, Baker E, Cherian S, Cutler S, Roynon-Reed A, Harrington K, Raithatha A, Bauchmuller K, Ahmad N, Grecu I, Trodd D, Martin J, Wrey Brown C, Arias AM, Craven T, Hope D, Singleton J, Clark S, Rae N, Welters I, Hamilton DO, Williams K, Waugh V, Shaw D, Puthucheary Z, Martin T, Santos F, Uddin R, Somerville A, Tatham KC, Jhanji S, Black E, Dela Rosa A, Howle R, Tully R, Drummond A, Dearden J, Philbin J, Munt S, Vuylsteke A, Chan C, Victor S, Matsa R, Gellamucho M, Creagh-Brown B, Tooley J, Montague L, De Beaux F, Bullman L, Kersiake I, Demetriou C, Mitchard S, Ramos L, White K, Donnison P, Johns M, Casey R,
Mattocks L, Salisbury S, Dark P, Claxton A, McLachlan D, Slevin K, Lee S, Hulme J, Joseph S, Kinney F, Senya HJ, Oborska A, Kayani A, Hadebe B, Orath Prabakaran R, Nichols L, Thomas M, Worner R, Faulkner B, Gendall E, Hayes K, Hamilton-Davies C, Chan C, Mfuko C, Abbass H, Mandadapu V, Leaver S, Forton D, Patel K, Paramasivam E, Powell M, Gould R, Wilby E, Howcroft C, Banach D, Fernández de Pinedo Artaraz Z, Cabreros L, White I, Croft M, Holland N, Pereira R, Zaki A, Johnson D, Jackson M, Garrard H, Juhaz V, Roy A, Rostron A, Woods L, Cornell S, Pillai S, Harford R, Rees T, Ivatt H, Sundara Raman A, Davey M, Lee K, Barber R, Chablani M, Brohi F, Jagannathan V, Clark M, Purvis S, Wetherill B, Dushianthan A, Cusack R, de Courcy-Golder K, Smith S, Jackson S, Attwood B, Parsons P, Page V, Zhao XB, Oza D, Rhodes J, Anderson T, Morris S, Xia Le Tai C, Thomas A, Keen A, Digby S, Cowley N, Wild L, Southern D, Reddy H, Campbell A, Watkins C, Smuts S, Touma O, Barnes N, Alexander P, Felton T, Ferguson S, Sellers K, Bradley-Potts J, Yates D, Birkinshaw I, Kell K, Marshall N, Carr-Knott L. Effect of ydrocortisone on Mortality and Organ Support in Patients With Severe COVID-19: The REMAP-CAP COVID-19 corticosteroid domain randomized clinical trial. JAMA. 2020;324(13):1317-1329. https://doi.org/10.1001/ jama.2020.17022.

11. Carey LA, Winer EP. I-SPY 2-toward more rapid progress in breast cancer treatment. N Engl J Med. 2016;375(1):83-4.

12. Goligher EC, Amato MBP, Slutsky AS. Applying precision medicine to trial design using physiology. Extracorporeal $\mathrm{CO}_{2}$ removal for acute respiratory distress syndrome. Am J Respir Crit Care Med. 2017;196(5):558-68.

13. Reddy K, Sinha P, O'Kane CM, Gordon AC, Calfee CS, McAuley DF. Subphenotypes in critical care: translation into clinical practice. Lancet Respir Med. 2020;8(6):631-43.

14. Angus DC. Optimizing the trade-off between learning and doing in a pandemic. JAMA J Am Med Assoc. 2020;323(19):1895-6.

15. Angus DC. Fusing randomized trials with big data: the key to self-learning health care systems? JAMA J Am Med Assoc. 2015;314(8):767-8.

\section{Publisher's Note}

Springer Nature remains neutral with regard to jurisdictional claims in published maps and institutional affiliations.
Ready to submit your research? Choose BMC and benefit from:

- fast, convenient online submission

- thorough peer review by experienced researchers in your field

- rapid publication on acceptance

- support for research data, including large and complex data types

- gold Open Access which fosters wider collaboration and increased citations

- maximum visibility for your research: over 100M website views per year

At BMC, research is always in progress.

Learn more biomedcentral.com/submissions 